\title{
Direct Lateral Minimally Invasive Approach for Excision of a L1/L2 Neurofibroma-A Technical Note and Literature Review
}

\author{
Soumya Mukherjee ${ }^{1} \quad$ James M. W. Robins ${ }^{1}$ \\ ${ }^{1}$ Department of Neurosurgery, Leeds General Infirmary, \\ Great George Street, Leeds, United Kingdom
}

Debasish Pal ${ }^{1}$

\author{
Abstract \\ Keywords \\ - spinal \\ - lateral approach \\ - minimally invasive \\ - neurofibroma \\ - retropleural \\ - retroperitoneal
}

\begin{abstract}
Address for correspondence James M. W. Robins, BMedSci (Hons), MBChB, MSc, MRCS, Department of Neurosurgery, Leeds General Infirmary, Leeds, LS1 3EX, United Kingdom (e-mail: jamesrobins@ nhs.net).
\end{abstract}

A case of resection of a large dumbbell-shaped nerve sheath tumor at L1/L2 via a direct lateral minimally invasive approach is described. The tumor was removed via a lateral, subdiaphragmatic, rib-sparing, retropleural, retroperitoneal approach. The thoracolumbar junction can be challenging via the lateral approach and to the best of our knowledge this is the first report of excision of a paravertebral tumor via this approach. We believe this provided the benefits of direct operative trajectory and exposure, excellent tumor access, and avoided the need for facetectomy and extended spinal instrumentation across the thoracolumbar junction. Complete tumor excision was achieved with preserved motor function and improvement in radicular pain and sensory neurological deficit.

\section{Introduction}

In this article, we describe the complete resection of a large L1/L2 extradural neurofibroma via a direct lateral minimally invasive approach. Access to the thoracolumbar junction can be challenging via the lateral approach due to many important adjacent structures that are vulnerable to damage. Although this approach is commonly described for degenerative pathology, here we report what we believe to be the first description of using this approach to resect a paravertebral tumor. The benefits we present of using this approach include direct operative trajectory and exposure, excellent tumor access, and crucially avoidance of the need for facetectomy. Avoidance of facetectomy prevents the development of instability that would necessitate extensive instrumentation across the transitional thoracolumbar junction.

In presenting this case, we hope to promote this technique of minimally invasive spinal tumor resection with less of the secondary effects that would be associated with an open procedure that, in turn, may be associated with greater patient morbidity.

\section{Case Report}

In this case, a 23-year-old woman presented with a 4-month history of right upper thigh burning pain and paraesthesia without weakness or sphincter disturbance. She had a past medical history of idiopathic aortic root dilatation. She had been recently started on regular pregabalin at an oral dose of $75 \mathrm{mg}$ twice daily for the right thigh neuropathic pain, otherwise she was not any regular medication. On neurological examination, she displayed reduced light touch sensation in the right L1 and L2 dermatomes, otherwise she had no other neurological deficit and her gait was normal. Magnetic resonance imaging (MRI) of the spine demonstrated a large $(5 \mathrm{~cm})$ right-sided tumor intimately related to the exiting right L1 nerve root without a central intradural component, consistent with a neurofibroma (-Fig. 1A). An initial radiological surveillance strategy demonstrated gradual interval growth in the tumor over 1 year. She was subsequently scheduled for surgery.

At surgery, the patient was positioned in the left lateral position, that is, right-side up, under general anesthetic. Electromyography (EMG) monitoring was utilized throughout the procedure. Intraoperative fluoroscopic C-arm radiographs and surface markings were made of the anterior and posterior borders of L1 and L2 vertebral bodies, the L1/L2 disc space, and the posterior border of the vertebral canal. An oblique linear incision was made over the palpable 12th rib, followed by careful dissection through the external oblique 
and internal oblique muscles. The 12th rib was bluntly stripped free of muscle fibers including the diaphragmatic attachment and periosteal layer elevated so that the rib was mobile (free-floating). Lung pleura was seen anteriorly and superiorly. This allowed for a subdiaphragmatic retroperitoneal approach down to the lateral spine toward the L1 transverse process. At this point, the large tumor was visible. An initial dilator (MaXcess System, NuVasive, Inc., San Diego, California, United States) was placed followed by the working retractor blades (with light source) around the tumor under continuous and directional EMG monitoring ( - Fig. 2A).
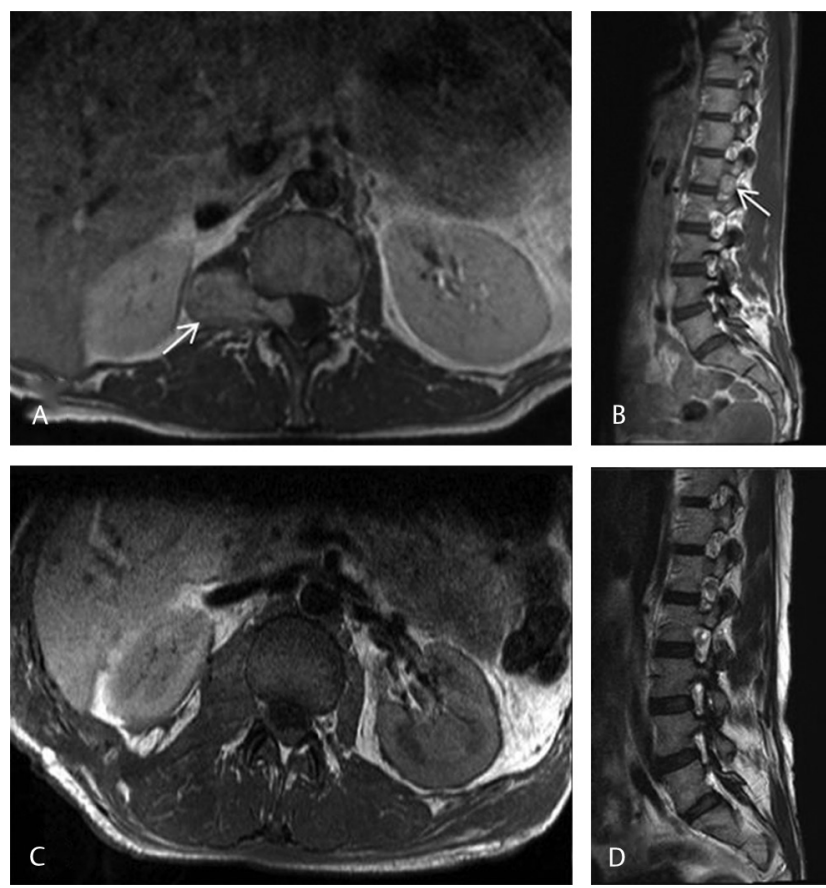

Fig. 1 Pre- and postoperative magnetic resonance imaging (MRI) of the right L1/L2 neurofibroma (A and $\mathbf{B}$ ) Preoperative axial and sagittal T1-weighted MRI postgadolinium demonstrating a large right-sided homogenously enhancing dumbbell-shaped nerve sheath tumor at L1/L2 extending into the foramina (white arrows) without a central intradural component. (C and $\mathbf{D}$ ) Forty-hour postoperative T1-weighted axial and sagittal MRI postgadolinium demonstrating complete tumor removal with minimal postoperative blood products within the resection cavity.
This provided a good surgical field and adequate access

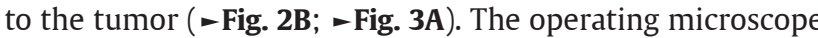
was then introduced. Using magnification and microinstruments, the superior and inferior poles of the tumor were defined and a circumferential tumor plane was established using standard microsurgical technique ( - Fig. 2C). Central intracapsular tumor debulking was then performed using a combination of rongeurs, bipolar diathermy, and cavitronic ultrasonic aspiration ( - Fig. 3B). At the inferior pole of initially debulked tumor, there was a visible nerve root confirmed on EMG monitoring and this was carefully separated off the tumor and preserved. The remaining tumor mass was removed in one piece. The wound was closed in layers. Just prior to the final suture to the muscle layer with the cavity just visible, some saline was irrigated into the wound and the anesthetist was requested to increase the intrapleural cavity pressure followed by quick insertion of the last stitch for closure of the muscle layer. This allowed the air to be driven out and wound closure without need of a chest drain. Neurophysiologic monitoring was unchanged throughout the procedure and total operative time was 95 minutes with $50 \mathrm{~mL}$ blood loss.

Postoperatively, the patient was ambulatory on the day of surgery and recovered uneventfully without any new neurological deficits. A routine postoperative chest radiograph showed a very small right apical pneumothorax, while the patient was asymptomatic, and this was managed conservatively, with complete radiological resolution after 48 hours. Two-day
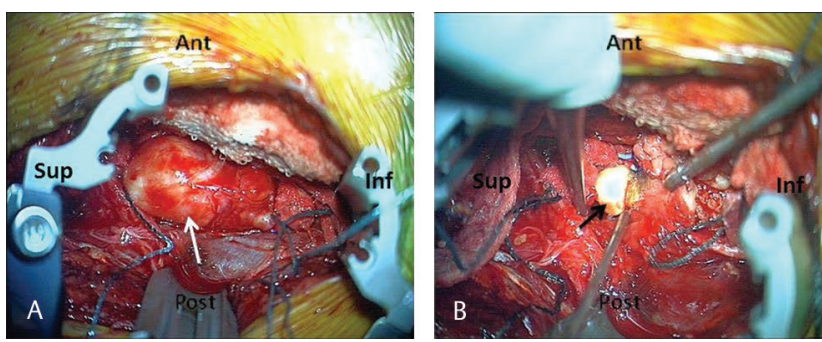

Fig. 3 Intraoperative photographs of the surgical field. (A) Retractor system docked around the tumor provided good access to the tumor (white arrow) with surgical patties placed at the superior and inferior poles. (B) Intracapsular tumor debulking (black arrow).
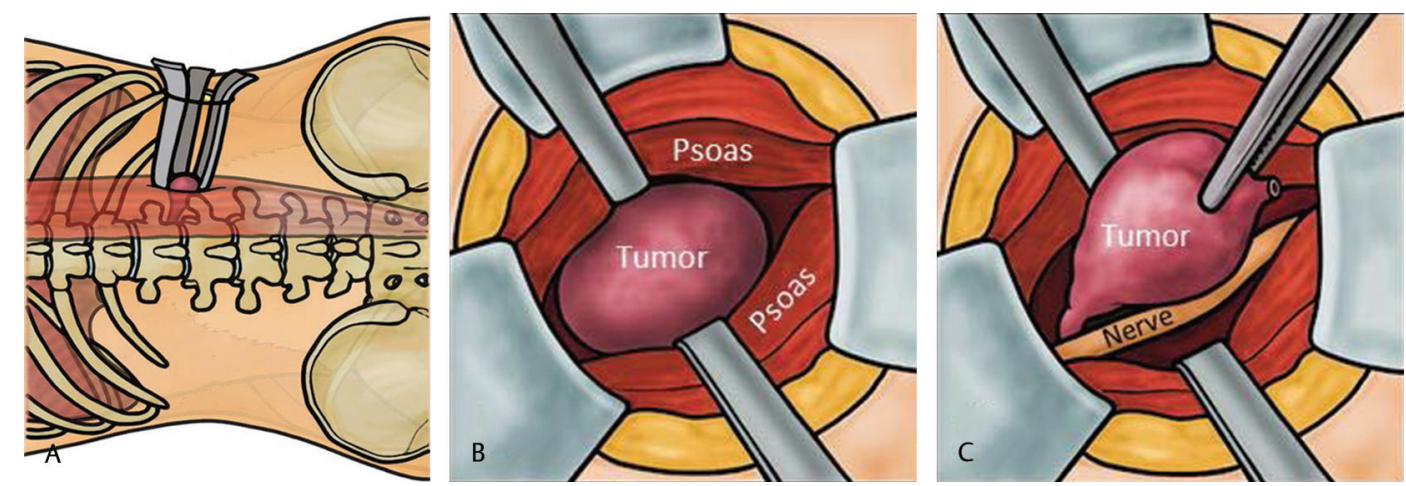

Fig. 2 Conceptual illustration of direct lateral, minimally invasive, rib sparing, subdiaphragmatic, retropleural, retroperitoneal approach in the left lateral position. (A) Superficial docking of tubular retractor within the psoas muscle. (B) Initial transpsoas tumor dissection. (C) Circumferential delineation of tumor from surrounding structures. 
postoperative MRI confirmed complete excision ( - Fig. 1B). She was discharged home 3 days following surgery. At 6-week clinic follow-up, her right-sided L1/L2 neuropathic pain had significantly diminished with improvement (although not resolution) in the numbness. Histopathology confirmed a World Health Organization (WHO) grade I neurofibroma.

\section{Discussion}

The direct lateral approach to the lumbosacral spine involves positioning the patient in the lateral decubitus position and careful muscle splitting dissection through the layers of external oblique, internal oblique and transversus abdominis, and subsequent opening of the transversalis fascia for retroperitoneal entry to the lateral spine. It was first described over a decade ago by Pimenta et al in 2006. ${ }^{1}$ It has since represented an increasingly accepted approach in the spine surgeon's armamentarium for a variety of indications, including interbody fusion. ${ }^{2}$ However, there are only a handful of case reports utilizing this technique for the resection of lumbosacral paravertebral and nerve sheath tumors and these have all been at lower lumbar levels (L3-L5). ${ }^{3-6}$ To the best of our knowledge, this is the first reported case describing a nuanced technique performed at the thoracolumbar junction at L1. The unique anatomic considerations at this junctional level include adjacent proximity of diaphragm and 12th rib superiorly, lung pleura anteriorly, and retroperitoneum posteriorly. Anatomical studies support the transthoracic approach and retroperitoneal approach for lesions above T12 and below the L1-L2 disc space, respectively. ${ }^{7}$ However, for lesions located between T12 vertebrae and the L1-L2 disc (as in this case), transthoracic, retroperitoneal, or retropleural approaches are all options, the most suitable of which depends on the condition being treated and the individual surgeon's experience. ${ }^{7}$

Our technique was subdiaphragmatic, rib-sparing, and retroperitoneal, achieved by careful muscular and periosteal elevation off the 12th rib to allow its mobilization for greater surgical access without the need for rib resection. The pleura was directly visualized anterosuperiorly and care taken to avoid its injury. At L1/L2, the psoas forms a thin muscular layer that is easily dissected with the contributors to the lumbar plexus, in particular the ilioinguinal and genitofemoral nerves, which pose a risk of injury (particularly the intrinsic motor branches) when spreading the muscle. This approach afforded direct visualization enabling identification of such branches and, together with careful superficial docking of the retractors and neurophysiologic (EMG) monitoring, minimized their risk of intraoperative injury.

Traditional approaches for resection of lumbar plexus and lateral nerve sheath tumors include the open or laparoscopic anterior retroperitoneal approach, which requires an access (general or vascular) surgeon and is technically demanding, necessitating very careful patient selection to avoid significant complications from damage to important adjacent structures. ${ }^{7}$ There are also paraspinal or midline posterior approaches. The latter requires significant muscle dissection extended laterally, facetectomy in particular for larger tumors (as in this case) to gain adequate access to the tumor, and potential compromise of joint stability requiring instrumented arthrodesis, ${ }^{8}$ which in this case would necessitate a longer construct (T12 or T10 to L2) across the thoracolumbar junctional level. In addition, all these approaches involve well-known risks such as hemorrhage, nerve injury, muscle injury as well as prolonged hospitalization for appropriate recovery. ${ }^{9}$

The technique described in this article presents a minimally invasive approach to paravertebral tumors at the L1 level, via a subdiaphragmatic, rib-sparing, retroperitoneal technique, enabling direct and full visualization of the tumor without the need for an access surgeon, the morbidity of a large open approach, or the difficulty of approaching a tumor deep to the psoas muscle microscopically. Gaining access to the tumor using this technique is relatively short (in this case within 25 minutes of the total 95-minute procedure) and is achieved here with minimal blood loss.

One limitation of this approach is that if there had been a more central intradural component to the tumor, this would have had to be debulked as a second-stage procedure via a posterior midline approach and would not have been adequately accessed by the lateral approach. This serves to emphasize the importance of careful case selection in planning the optimal surgical route. Even if a second procedure to deal with an intradural component would have been necessary, this would have been far less invasive as compared with a single stage posterior approach. The other main disadvantages of this technique are the additional risks of pleural injury causing pneumothorax (in our case this was small and resolved with conservative management), incisional hernia formation (use of a tubular retractor as in our case affords smaller incisions reducing the risk of incisional hernia), or lumbar plexus injury (EMG monitoring as utilized in this case reduces this risk).

\section{Conclusion}

Here we present the usefulness of the direct lateral minimally invasive approach for excision of neurofibromas (in particular lateral tumors without a significant intradural portion) particularly at or near the thoracolumbar junction. This approach provided the benefits of direct operative trajectory and exposure, excellent access to the tumor, shorter operative time with minimal blood loss, and avoidance of the need for facetectomy and extended spinal instrumentation across the thoracolumbar junction. This approach achieved an excellent postoperative outcome in terms of complete excision and resolution of neuropathic pain and numbness.

\section{Note}

S. M. is a senior trainee in Neurosurgery with a specialist interest in spinal surgery. J. M. W. R. is a trainee in Neurosurgery with a specialist interest in spinal surgery. D. P. is a senior Consultant Neurosurgeon with subspecialty expertise in spinal surgery. 


\section{Authors' Contributions}

S. M. was involved in performing the operation, collecting patient data including images, literature review, and writing of the manuscript, and revised it for intellectual content. J. M. W. R. was involved in collecting patient data, literature review, and writing of the manuscript, and revised it for intellectual content. D. P. performed the operation as the lead consultant neurosurgeon, and significantly contributed to writing of the manuscript, and revised it for intellectual content. All authors read and approved the final manuscript.

\section{Funding}

None.

\section{Conflict of Interest}

None declared.

\section{References}

1 Ozgur BM, Aryan HE, Pimenta L, Taylor WR. Extreme Lateral Interbody Fusion (XLIF): a novel surgical technique for anterior lumbar interbody fusion. Spine J 2006;6(4):435-443

2 Xu DS, Walker CT, Godzik J, Turner JD, Smith W, Uribe JS. Minimally invasive anterior, lateral, and oblique lumbar interbody fusion: a literature review. Ann Transl Med 2018;6(6):104

3 Boah AO, Perin NI. Lateral access to paravertebral tumors. J Neurosurg Spine 2016;24(5):824-828
4 Benjamin CG, Oermann EK, Thomas JA, Distaso CT, Sandhu FA. Minimally invasive direct lateral transpsoas approach for the resection of a lumbar plexus schwannoma: technique report. Surg J (N Y) 2016;2(3):e66-e69

5 Safaee MM, Ames CP, Deviren V, Clark AJ. Minimally invasive lateral retroperitoneal approach for resection of extraforaminal lumbar plexus schwannomas: operative techniques and literature review. Oper Neurosurg (Hagerstown) 2018;15(5):516-521

6 Shah M, Kaminsky J, Vougioukas VI. Minimally invasive removal of an extradural intraradicular lumbar schwannoma. Acta Neurochir (Wien) 2008;150(7):691-693, discussion 693-694

7 Sun JC, Wang JR, Luo T, et al. Surgical incision and approach in thoracolumbar extreme lateral interbody fusion surgery: an anatomic study of the diaphragmatic attachments. Spine 2016;41(4):E186-E190

8 Nishio A, Adachi W, Igarashi J, Koide N, Kajikawa S, Amano J. Laparoscopic resection of a retroperitoneal schwannoma. Surg Laparosc Endosc Percutan Tech 1999;9(4):306-309

9 O'Toole JE, Eichholz KM, Fessler RG. Minimally invasive approaches to vertebral column and spinal cord tumors. Neurosurg Clin N Am 2006;17(4):491-506 